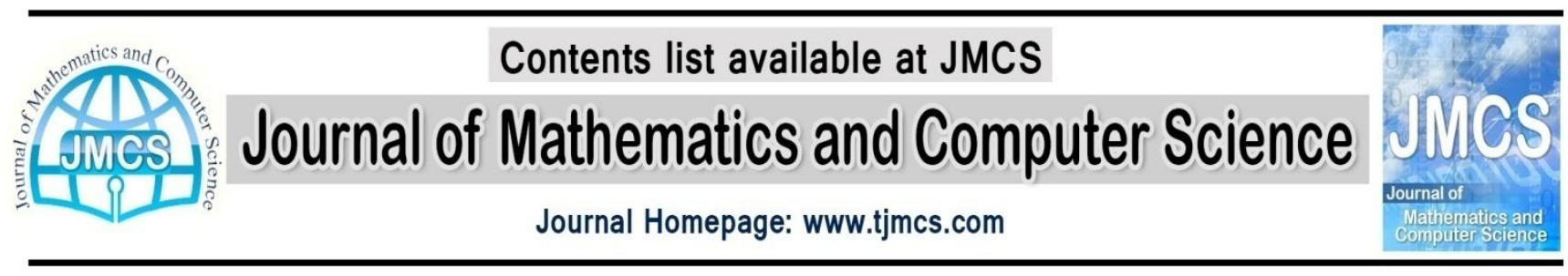

\title{
Numerical Solution for Random Forced SPDE via Galerkin Finite Element Method
}

\author{
Rasoul Naseri, A. Malek ${ }^{1}$ \\ Department of Applied Mathematics, Faculty of Mathematical Sciences, Tarbiat Modares University, \\ P. O. Box 14115-134, Tehran, Iran \\ (naserir@modares.ac.ir\& mala@modares.ac.ir) \\ Article history: \\ Received October 2013 \\ Accepted December 2013 \\ Available online December 2013
}

\begin{abstract}
In this paper, we present a deterministic finite element approach for solving a random forced Diffusion equation. Separation of random and deterministic variables is done by KarhunenLoeve expansion. Truncating the Karhunen-Loeve expansion of the permeability field leads to a finite dimensional approximation of the problem. The problem is discretized, in spatial part, using the finite-element method and the polynomial chaos expansion in stochastic part. Finally, using Kronecker product preconditioner and thus, preconditioned conjugate gradient method the governed system of equation is solved. Numerical experiments are presented for illustrating the theoretical results.
\end{abstract}

Keywords: Stochastic partial differential equation, Karhunen-Loeve expansion, Wiener Chaos expansion, finite element method.

\section{Introduction}

Physical problems, often, can be formulated as mathematical models which in many cases contain partial differential equations (PDEs). Uncertainty might plagues everything from modeling assumptions to experimental data. So, the differential coefficient and source functions in the model might represent as functions of the spatial domain and some sample space. In this case we deal with a stochastic partial differential equation (SPDE). In this paper, we consider the stochastic steady-state diffusion equation along with homogeneous Dirichlet boundary-value conditions. In stochastic engineering, the perturbation method is a popular technique, cf., e.g., [7, 10, 11, 13, 14, 16, 21]. Considering certain smoothness conditions, the random functions and operators involved in the differential equation are expanded in a Taylor series about their respective mean values. In this way, usually good results are obtained only for small deviations, cf., e.g., [11,16]. The Neumann expansion series method is another approach. In this

\footnotetext{
${ }^{1}$ Corresponding author.
}

E-mail address: mala@modares.ac.ir. 
approach, the inverse of the stochastic operator is approximated by its Neumann series [4,18]. Recently, a method based on a spectral representation of the uncertainty is introduced by Ghanem and Spanos [9], where utilizes the Karhunen-Loeve expansion (KLE) of correlated random functions, cf. [12]. In order to allow for other representations of the random processes, the spectral finite element scheme is generalized by Babuska and et al. [1,2,3]. Here, we follow the Galerkin finite element method of stochastic diffusion problems. We use the kronecker product preconditioner presented by Ullmann [20] to clustering the eigenvalues of the global matrix gained at the end of variational computation. Thus, using preconditioned conjugate gradient method [17], the large scale system of equation is solved, which gives good computational results. The organization of this paper is as follow: In Section 2, Karhunen-Loeve expansion (KLE) and polynomial chaos expansion (PCE) is presented. In Section 3, stochastic Galerkin method, kroneker product preconditioner and computation of statistics is proposed. In Section 4, some numerical result is illustrated.

\section{Problem formulation}

Our model problem is a steady state diffusion problem in a $2 D$ domain with inhomogeneous stochastic diffusion coefficient $a(x, \omega)$ :

$$
\begin{aligned}
\nabla \cdot(a(x, \omega) \nabla u(x, \omega)) & =z(x, \omega) \quad \text { in } D \times \Omega \\
u(x, \omega) & =0
\end{aligned}
$$

where $D$ is the spatial domain, $\Omega$ is probability space and $a(x, \omega)$ is the correlated random fields. Hence, the solutions $u(x, \omega)$ of the SPDE (1) are also random fields. As an important assumption for the stochastic diffusion equation (1), it is assumed that the random coefficient $u(x, \omega)$ satisfies the elliptic condition. That is, there exist a constant $a_{\text {min }}$ such that

$$
0<a_{\min } \leq a(x, \omega)
$$

\subsection{Karhunen-Loeve Expansion (KLE)}

Consider a random field $a(x, \omega), x \in D$, with finite second order moment

$$
\int_{\Omega} E\left[a^{2}(x, \omega)\right] d x<\infty
$$

Assume that $E[a]=\bar{a}(x)$. It is possible to expand $a(x, \omega)$, for a given orthonormal basis $\left\{\psi_{k}\right\}$ in $L^{2}(D)$, as a generalized Fourier series

where

$$
a(x, \omega)=\bar{a}(x)+\sum_{k=1}^{\infty} a_{k}(\omega) \psi_{k}(x)
$$

$$
a_{k}(\omega)=\int_{\Omega} a(x, \omega) \psi_{k}(x) d x, \quad k=1,2, \ldots
$$

are random variables with zero means. It is important, now, to find a special basis $\left\{\phi_{k}\right\}$ that makes corresponding $a_{k}$ uncorrelated: $E\left[a_{i} a_{j}\right]=0$ for al $1 i \neq j$. Denoting the covariance function of $a(x, \omega)$ by $R(x, y)=E[a(x, \omega) a(y, \omega)]$, the basis functions $\left\{\phi_{k}\right\}$ should satisfy 


$$
E\left[a_{i} a_{j}\right]=\int_{D} \phi_{i}(x) d x \int_{D} R(x, y) \phi_{j}(y) d y=0, \quad i \neq j
$$

Complete and orthonormality of $\left\{\phi_{k}\right\}$ in $L^{2}(D)$ follows that $\phi_{k}(x)$ are eigenfunctions of $R(x, y)$ :

$$
\int_{D} R(x, y) \phi_{j}(\boldsymbol{y}) d y=\lambda_{j} \phi_{j}(x), \quad j=1,2, \ldots
$$

where $\lambda_{j}=E\left[a_{j}^{2}\right]>0$. Indeed, by choosing basis functions $\phi_{k}(x)$ as the solutions of the eigenproblem (4), the random variables $a_{k}(\omega)$ will be uncorrelated. Denoting $\theta_{k}=a_{k} / \sqrt{\lambda_{k}}$, we have the following expansion:

$$
a(x, \omega)=\bar{a}(x)+\sum_{k=1}^{\infty} \sqrt{\lambda_{k}} \theta_{k}(\omega) \phi_{k}(x)
$$

where $\theta_{k}$ satisfy $E\left[\theta_{k}\right]=0$ and $E\left[\theta_{i} \theta_{j}\right]=\delta_{i j}$. In the case that $a(x, \omega)$ is considered as a Gaussian process, $a_{k}(\omega), k=1,2, \ldots$ will be independent Gaussian random variables. The expansion (5) is known as the Karhunen-Loeve expansion (KLE) of the stochastic process $a(x, \omega)$.

Using the KLE (5), the stochastic process can be represented as a series of uncorrelated random variables. Since the basic functions $\phi_{k}(\boldsymbol{x})$ are deterministic, the spatial dependence of the random process can be resolved by them. The KLE converges to the random process $a(x, \omega)$ in the mean square sense

$$
\lim _{N \rightarrow \infty} \int_{D} E\left|a(x, \omega)-a_{N}(x, \omega)\right|^{2} d x=0
$$

where

$$
a_{N}=\bar{a}(x)+\sum_{k=1}^{N} \sqrt{\lambda_{k}} \theta_{k} \phi_{k}
$$

is a finite term [12,15]. So, among all the Fourier expansions (3), the KLE with the basis function $\phi_{k}(x)$ determined by the eigen problem (4) has the fastest convergence rate. The convergence rate of the KLE only depends on the smoothness of the covariance function, but not on the specific probability structure of the process being expanded. The KLE converges fast if the covariance function $R(x, y)$ is very smooth. In this case, we only need to keep the leading order terms (quantified by the magnitude of $\lambda_{k}$ ) in the finite KLE and still capture most of the energy of the stochastic process $a(x, \omega)$. For the finite KLE (6), we define its energy ratio as

$$
e(N)=\frac{\int_{\Omega} E\left|a_{N}\right|^{2} d x}{\int_{\Omega} E|a|^{2} d x}=\frac{\sum_{k=1}^{N} \lambda_{k}}{\sum_{k=1}^{\infty} \lambda_{k}}
$$

If the eigenvalues $\lambda_{k}, k=1,2, \ldots$, decay very fast, then the finite term KLE would be good approximation of the stochastic process $[5,8]$.

\subsection{Polynomial Chaos Expansion (PCE)}

There are problems, as the solution of a PDE with random inputs, that the covariance function of a random process $u(x, \omega), x \in D$ is not known. The solution of such problems can be represented using a polynomial chaos expansion (PCE) given by

$$
u(x, \omega)=\sum_{k=1}^{\infty} u_{k}(x) \Psi_{k}(\omega)
$$


where, the functions $u_{k}(x)$ are deterministic coefficients, $\omega$ is a vector of orthonormal random variables and $\Psi_{k}(\omega)$ are multi-dimensional orthogonal polynomials with the following properties:

$$
\left\langle\Psi_{1}\right\rangle \equiv E\left[\Psi_{1}\right]=1, \quad\left\langle\Psi_{k}\right\rangle=0, k>1, \quad\left\langle\Psi_{i} \Psi_{j}\right\rangle=h_{i} \delta_{i j}
$$

The convergence property of PCE for a random quantity in $L^{2}$ is ensured by the Cameron-Martin theorem $[5,22]$, i.e.

$$
\left\langle u(x, \omega)-\sum_{k=1}^{\infty} u_{k}(x) \Psi_{k}(\omega)\right\rangle \stackrel{L^{2}}{\rightarrow} 0 .
$$

Hence, for the sake of computation, this convergence justifies a truncation of PCE to a finite number of terms,

$$
u(x, \omega)=\sum_{k=1}^{P} u_{k}(x) \Psi_{k}(\omega)
$$

where, the value of $P$ is determined by the highest degree of polynomial $d$, used to represent $u$, and the number $N$ of random variables - the length of $\omega$ - with the formula $P+1=(N+d)$ !/ $N$ ! $d$ ! [22]. Generally, the value of $N$ is the same as the number of uncorrelated random variables in the system or equivalently, the truncation length of the truncated KLE. Typically, the value of $d$ is chosen by some heuristic method. Indeed, in the case of $d=1$ and $N$ random variables, the KLE is a special case of the PCE.

\section{Stochastic Galerkin}

Suppose $W_{i} \subset L_{\rho_{i}}^{2}\left(\Omega_{i}\right)$ with dimension $\rho_{i}$ for $i=1, \ldots, M$ and $V \subset H_{0}^{l}(D)$ with dimension $N$. In addition; let $\left\{\psi_{n}^{i}\right\}_{n=1}^{p_{i}}$ for $i=1, \ldots, M$ be basis of $W_{i}$ and $\left\{\phi_{i}\right\}_{i=1}^{N}$ a basis of $V$. The finite dimensional tensor product space $W_{1} \otimes \ldots \otimes W_{M} \otimes V$ can be defined as the space spanned by the functions $\left\{\psi_{n_{1}}^{1}, \ldots, \psi_{n_{M}}^{M} \phi_{i}\right\}$ for all $n_{1} \in\left\{1, \ldots, p_{1}\right\}, \ldots, n_{M} \in\left\{1, \ldots, p_{M}\right\}$ and $i \in\{1, \ldots, N\}$. To simplify notation, let $\mathbf{n}$ denote a multiindex whose $k$ th component $n_{k} \in\left\{1, \ldots, p_{k}\right\}$ and $I$ denote the set of such multi-indices, then the basis functions for the tensor product space have the form

where

$$
v_{\mathbf{n} i}(x, \omega)=\phi_{i}(x) \Psi_{\mathrm{n}}(\omega)
$$

$$
\Psi_{\mathrm{n}}=\prod_{k=1}^{M} \psi_{n_{k}}^{k}\left(\omega_{k}\right)
$$

Then, the Galerkin method is looking for a solution $\hat{u} \in W_{l} \otimes \ldots \otimes W_{M} \otimes V$ such that

$$
\int_{\Omega} \rho(\omega) \int_{D} a(x, \omega) \nabla u_{h P}(x, \omega) \cdot \nabla v_{\mathbf{n} i}(x, \omega) d x d \omega=\int_{\Omega} \rho(\omega) \int_{D} z(x, \omega) v_{\mathbf{n} i}(x, \omega) d x d \omega
$$

for all $\mathbf{n} \in \boldsymbol{I}$ and $i=1, \ldots, N$, which $\rho(\omega)$ is the integration weight. Equivalently,plugging in the explicit formula for the basic functions, equation (10) can be written as

$$
\int_{\Omega} \rho(\omega) \Psi_{\mathbf{n}}(\omega) \int_{D} a(x, \omega) \nabla u_{h P}(x, \omega) \cdot \nabla \phi_{i}(x) d x d \omega=\int_{\Omega} \rho(\omega) \Psi_{\mathbf{n}}(\omega) \int_{D} z(x, \omega) \phi_{i}(x) d x d \omega
$$

for all $\mathbf{n} \in \boldsymbol{I}$ and $i=1, \ldots, N$. Now, considering the approximate solution as 


$$
u_{h P}(x, \omega)=\sum_{j=1}^{N} \sum_{\mathbf{m} \in \boldsymbol{I}} u_{j \mathbf{m}} \phi_{j}(x) \Psi_{\mathbf{m}}(\omega)
$$

and substituting (12) into the equation (11) yields

$$
\begin{aligned}
& \sum_{j=1}^{N} \sum_{\mathbf{m} \in \boldsymbol{I}} u_{j \mathbf{m}} \int_{\Omega} \rho(\omega) \Psi_{\mathbf{n}}(\omega) \Psi_{\mathbf{m}}(\omega) \int_{\mathrm{D}} a(x, \omega) \nabla \phi_{j}(x) \cdot \nabla \phi_{i}(x) d x d \omega \\
& =\int_{\Omega} \rho(\omega) \Psi_{\mathbf{n}}(\omega) \int_{D} z(x, \omega) \phi_{i}(x) d x d \omega
\end{aligned}
$$

for all $\mathbf{m} \in \boldsymbol{I}$ and $i=1, \ldots, N$. Let $P=|\boldsymbol{I}|=\prod_{\mathrm{k}=1}^{\mathrm{M}} p_{k}$, then, a natural bijection between $\{1, \ldots, P\}$ and $\boldsymbol{I}$ can be defined. Thus, equation (13) can be written as

$$
\begin{gathered}
\sum_{j=1}^{N} \sum_{m=1}^{P} u_{j m} \int_{\Omega} \rho(\omega) \Psi_{n}(\omega) \Psi_{m}(\omega) \int_{\mathrm{D}} a(x, \omega) \nabla \phi_{j}(x) \cdot \nabla \phi_{i}(x) d x d \omega \\
=\int_{\Omega} \rho(\omega) \Psi_{n}(\omega) \int_{D} z(x, \omega) \phi_{i}(x) d x d \omega
\end{gathered}
$$

Now, if $a$ and $z$ have the following KLE:

and

$$
a(x, \omega)=a_{0}(x)+\sum_{i=1}^{M} y_{i} a_{i}(x)
$$

$$
z(x, \omega)=z_{0}(x)+\sum_{i=1}^{M} y_{i} z_{i}(x)
$$

Then, we can rewrite equation (14) as

$$
\begin{gathered}
\sum_{j=1}^{N} \sum_{m=1}^{P} u_{j m}\left(\left(K_{0}\right)_{i, j} \int_{\Omega} \rho(\omega) \Psi_{n}(\omega) \Psi_{m}(\omega) d \omega+\sum_{m=1}^{P}\left(K_{k}\right)_{i, j} \int_{\Omega} \rho(\omega) \Psi_{n}(\omega) \Psi_{m}(\omega) y_{k} d \omega\right) \\
=\left(z_{0}\right)_{i} \int_{\Omega} \rho(\omega) \Psi_{n}(\omega) d \omega+\sum_{m=1}^{P}\left(z_{k}\right)_{i} \int_{\Omega} \rho(\omega) \Psi_{n}(\omega) y_{k} d \omega
\end{gathered}
$$

where

$$
\left(K_{k}\right)_{i, j}=\int_{D} a_{k}(x) \nabla \phi_{i}(x) \cdot \nabla \phi_{j}(x) d x \cong \sum_{r} w_{r} a_{k}\left(x_{r}\right) \nabla \phi_{i}\left(x_{r}\right) \cdot \nabla \phi_{j}\left(x_{r}\right)
$$

and

$$
\left(z_{k}\right)_{i}=\int_{D} z_{i}(x) \phi_{i}(x) d x \cong \sum_{r} w_{r} z_{i}\left(x_{r}\right) \phi_{i}\left(x_{r}\right)
$$

for $k=1, \ldots, M$ and $i=1, \ldots, N$ [3]. Equations (16) and (17) are approximated by quadrature rule in spatial domain. Now, assume that there exist functions $\Psi_{n}, n=1, \ldots, P$ such that 


$$
\int_{\Omega} \rho(\omega) \Psi_{n}(\omega) \Psi_{m}(\omega) d \omega=\delta_{m n}
$$

and

$$
\int_{\Omega} \rho(\omega) \Psi_{n}(\omega) \Psi_{m}(\omega) y_{k} d \omega=C_{k n} \delta_{m n}
$$

where, for $m=n$, the above integral is approximated using quadrature rule in random domain as follow:

$$
\int_{\Omega} \rho(\omega) \Psi_{n}(\omega) \Psi_{n}(\omega) y_{k} d \omega \cong \sum_{s} w_{s}^{\prime} \rho\left(\omega_{s}\right) \Psi_{n}^{2}\left(\omega_{s}\right) y_{k}\left(\omega_{s}\right)
$$

Then, equation (15) becomes

$$
\begin{aligned}
& \sum_{j=1}^{N} \sum_{m=1}^{P} u_{j m}\left(\left(K_{0}\right)_{i, j}+\sum_{k=1}^{M} C_{k n}\left(K_{k}\right)_{i, j}\right) \delta_{m n} \\
& \quad=\left(z_{0}\right)_{i} \int_{\Omega} \rho(\omega) \Psi_{n}(\omega) d \omega+\sum_{k=1}^{M}\left(z_{k}\right)_{i} \int_{\Omega} \rho(\omega) \Psi_{n}(\omega) y_{k} d \omega
\end{aligned}
$$

or, equivalently,

$$
\sum_{j=1}^{N} u_{m j}\left(\left(K_{0}\right)_{i, j}+\sum_{k=1}^{M} C_{k n}\left(K_{k}\right)_{i, j}\right)=\left(z_{0}\right)_{i} \int_{\Omega} \rho(\omega) \Psi_{n}(\omega) d \omega+\sum_{k=1}^{M}\left(z_{k}\right)_{i} \int_{\Omega} \rho(\omega) \Psi_{n}(\omega) y_{k} d \omega
$$

In equations (19) and (20) we have:

$$
\int_{\Omega} \rho(\omega) \Psi_{n}(\omega) d \omega=0 ; \quad \text { for } n \neq 0,
$$

and

$$
\int_{\Omega} \rho(\omega) \Psi_{n}(\omega) y_{k} d \omega=\int_{\Omega} \rho(\omega) \Psi_{0}(\omega) \Psi_{n}(\omega) y_{k} d \omega=C_{k n} \delta_{0 n}=C_{k 0}
$$

Finally, equation (20) can be considered as the following block diagonal system:

$$
\left[\begin{array}{ccc}
K_{0}+\sum_{k=1}^{P} C_{k 1} K_{k} & \cdots & 0 \\
\vdots & \ddots & \vdots \\
0 & \cdots & K_{0}+\sum_{k=1}^{P} C_{k p} K_{k}
\end{array}\right]\left(\begin{array}{c}
\vec{u}_{1} \\
\vdots \\
\vec{u}_{P}
\end{array}\right)=\left(\begin{array}{c}
\vec{z}_{1} \\
\vdots \\
\vec{z}_{p}
\end{array}\right)
$$

or briefly, $K \vec{u}=\vec{z}$. Once $u$ has been computed, it can be post-processed to obtain meaningful information, such as the mean and variance of $u_{h P}$. Definitions of mean and variance of a random field (noting that a random field is a stochastic process) can be found in $[14,19]$. The expected value of $u_{h P}$ is given by

$$
E\left(u_{h P}\right)=\int_{\Omega} \rho(\omega)\left(\sum_{j=1}^{N} \sum_{m=1}^{P} u_{j m} \phi_{j}(x) \Psi_{\mathrm{m}}(\omega)\right) d \omega
$$




$$
\begin{gathered}
=\sum_{j=1}^{N} \sum_{m=1}^{P} u_{j m} \phi_{j}(x) \int_{\Omega} \rho(\omega) \Psi_{\mathrm{m}}(\omega) d \omega \\
=\sum_{j=1}^{N} u_{j 1} \phi_{j}(x)
\end{gathered}
$$

on account that $\Psi_{1}=1$ and the orthogonality conditions (8). Therefore, if $x_{j}$ is a node corresponding to the basis function $\phi_{j}, E\left(u_{h P}\left(x_{j}, \omega\right)\right)=u_{j l}$, the variance of $u_{h P}$ is given by

$$
\begin{aligned}
V\left(u_{h P}\right)=\int_{\Omega} \rho(\omega)\left(\sum_{j=1}^{N} \sum_{m=1}^{P} u_{j m} \phi_{j}(x) \Psi_{\mathrm{m}}(\omega)\right)^{2} d \omega-\left(E\left(u_{h P}\right)\right)^{2} \\
=\sum_{j=1}^{N} \sum_{m=1}^{P} u_{j m}^{2} \phi_{j}^{2}(x) \int_{\Omega} \rho(\omega) \Psi_{\mathrm{m}}^{2}(\omega) d \omega-\left(\sum_{j=1}^{N} u_{j l} \phi_{j}(x)\right)^{2}
\end{aligned}
$$

Adopting the notation

$$
u_{k}(x)=\sum_{j=1}^{N}\left(\vec{u}_{k}\right)_{j} \phi_{j}(x)
$$

one can compute the $m^{\text {th }}$ statistical moments of the solution as

$$
E\left[u(., x)^{m}\right] \approx E\left[\left(\sum_{k=1}^{P} \Psi_{k}(.) u_{k}(x)\right)^{m}\right]=\sum_{k=1}^{P} \ldots \sum_{k=1}^{P} E\left[\Psi_{k_{1}}(.) \ldots \Psi_{k_{m}}\right] u_{k_{1}}(x) \ldots u_{k_{m}}(x) .
$$

\subsection{Kronecker product preconditioners.}

Rewriting equation (21) as:

$$
\underbrace{\left(\sum_{k=0}^{M} \mathrm{G}_{\mathrm{k}} \otimes K_{k}\right)}_{\overparen{R}} \vec{u}=\vec{z}
$$

the strategy is to choose $\widehat{\mathrm{P}}=G \otimes K_{0}$ as a preconditioner, such that

$$
G=\operatorname{argmin}\left\{H \in \mathbb{R}^{\mathrm{P} \times \mathrm{P}}:\left\|K-\mathrm{H} \otimes K_{0}\right\|_{F}\right\}
$$

where, as mentioned in PCE, $\mathrm{P}$ is equals with the degree of polynomial chaos truncation, and $\|.\|_{F}$ denotes the Frobenius norm. The closed form of the solution can be written as follow [6,20],

$$
G=I+\sum_{P} \frac{\operatorname{tr}\left(K_{k}^{T} K_{0}\right)}{\operatorname{tr}\left(K_{0}^{T} K_{0}\right)} G_{k}
$$

Which $\operatorname{tr}\left(K_{k}^{T} K_{0}\right)=\sum_{i=1}^{N_{q}}\left[K_{k}\right]_{i, i}\left[K_{0}\right]_{i, i}$ and hence, the coefficients in above equality can be computed straightforward. In addition, since $K$ and $K_{0}$ are symmetric and positive definite, so $G$ and $\hat{P}=G \otimes K_{0}$ have also these properties. Now, using preconditioned Newton's conjugate gradient algorithm, we can find fast and accurate convergence of the solution [20].

In fact $\hat{P}$ can be written as: 


$$
\hat{P}=\left[\begin{array}{ccc}
1+\sum_{k=1}^{M} C_{k 1} \frac{\operatorname{tr}\left(K_{k}^{T} K_{0}\right)}{\operatorname{tr}\left(K_{0}^{T} K_{0}\right)} & \cdots & 0 \\
\vdots & \ddots & \vdots \\
0 & \cdots & 1+\sum_{k=1}^{M} C_{k p} \frac{\operatorname{tr}\left(K_{k}^{T} K_{0}\right)}{\operatorname{tr}\left(K_{0}^{T} K_{0}\right)}
\end{array}\right]
$$

So $\hat{P}^{-1} K$ can be expressed as:

$$
\hat{P}^{-1} K=\left[\begin{array}{ccc}
\frac{K_{0}+\sum_{k=1}^{M} C_{k 1} K_{k}}{1+\sum_{k=1}^{M} C_{k 1} \frac{\operatorname{tr}\left(K_{k}^{T} K_{0}\right)}{\operatorname{tr}\left(K_{0}^{T} K_{0}\right)}} & \cdots & 0 \\
\vdots & \ddots & \vdots \\
0 & \cdots & \frac{K_{0}+\sum_{k=1}^{M} C_{k p} K_{k}}{1+\sum_{k=1}^{M} C_{k p} \frac{\operatorname{tr}\left(K_{k}^{T} K_{0}\right)}{\operatorname{tr}\left(K_{0}^{T} K_{0}\right)}}
\end{array}\right]
$$

Finally, after preconditioning the problem, we are interested in solving the following equation:

$$
\left[\begin{array}{ccc}
\frac{K_{0}+\sum_{k=1}^{M} C_{k 1} K_{k}}{1+\sum_{k=1}^{M} C_{k 1} \frac{\operatorname{tr}\left(K_{k}^{T} K_{0}\right)}{\operatorname{tr}\left(K_{0}^{T} K_{0}\right)}} & \cdots & 0 \\
\vdots & \ddots & \vdots \\
0 & \cdots & \frac{K_{0}+\sum_{k=1}^{M} C_{k p} K_{k}}{1+\sum_{k=1}^{M} C_{k 1} \frac{\operatorname{tr}\left(K_{k}^{T} K_{0}\right)}{\operatorname{tr}\left(K_{0}^{T} K_{0}\right)}}
\end{array}\right]\left(\begin{array}{c}
\overrightarrow{\mathrm{u}}_{1} \\
\vdots \\
\overrightarrow{\mathrm{u}}_{\mathrm{P}}
\end{array}\right)=\left(\begin{array}{c}
\frac{\overrightarrow{\mathrm{z}}^{1}}{1+\sum_{k=1}^{M} C_{k 1} \frac{\operatorname{tr}\left(K_{k}^{T} K_{0}\right)}{\operatorname{tr}\left(K_{0}^{T} K_{0}\right)}} \\
\vdots \\
\overrightarrow{\mathrm{z}}^{\mathrm{P}} \\
1+\sum_{k=1}^{M} C_{k 1} \frac{\operatorname{tr}\left(K_{k}^{T} K_{0}\right)}{\operatorname{tr}\left(K_{0}^{T} K_{0}\right)}
\end{array}\right)
$$

Using Newton's conjugate gradient algorithm we can find fast and accurate convergence of the solution.

\section{Experimental results}

Example 1- 2D Poisson Equation: The first example is a Poisson equation in two spatial dimensions with one constant random parameter. Let $D=\{(x, y):-1 \leq x \leq 1,-1 \leq y \leq 1\}$ be the spatial domain of the problem and $\Omega$ the sample space. Then we seek a solution $u(x, y, \omega)$ such that satisfies

$$
\begin{gathered}
a(\omega)\left(u(x, y, \omega)_{x x}+u(x, y, \omega)_{y y}\right)=\exp \left[-64 *\left(\left(x-\frac{1}{2}\right)^{2}+\left(y-\frac{1}{2}\right)^{2}\right)\right] \text { in } D \times \Omega \\
u(x, y, \omega)=0 \\
\text { on } D \times \partial \Omega
\end{gathered}
$$

where $a(\omega)$ is a random variable distributed uniformly over the interval [1,3]. First we express $a$ in terms of a uniform random variable on $[-1,1]$, i.e.

$$
a=\xi+2 \quad \text { where } \xi \sim U[-1,1] \text {. }
$$

Figure 1, illustrates approximated solution, standard deviation and expectation counter plot and global matrix sparsity, for $50 * 50$ mesh points of spatial domain, $\mathrm{d}=3$, (number of random variables) and $\mathrm{N}=4$ (degree of polynomials) . 

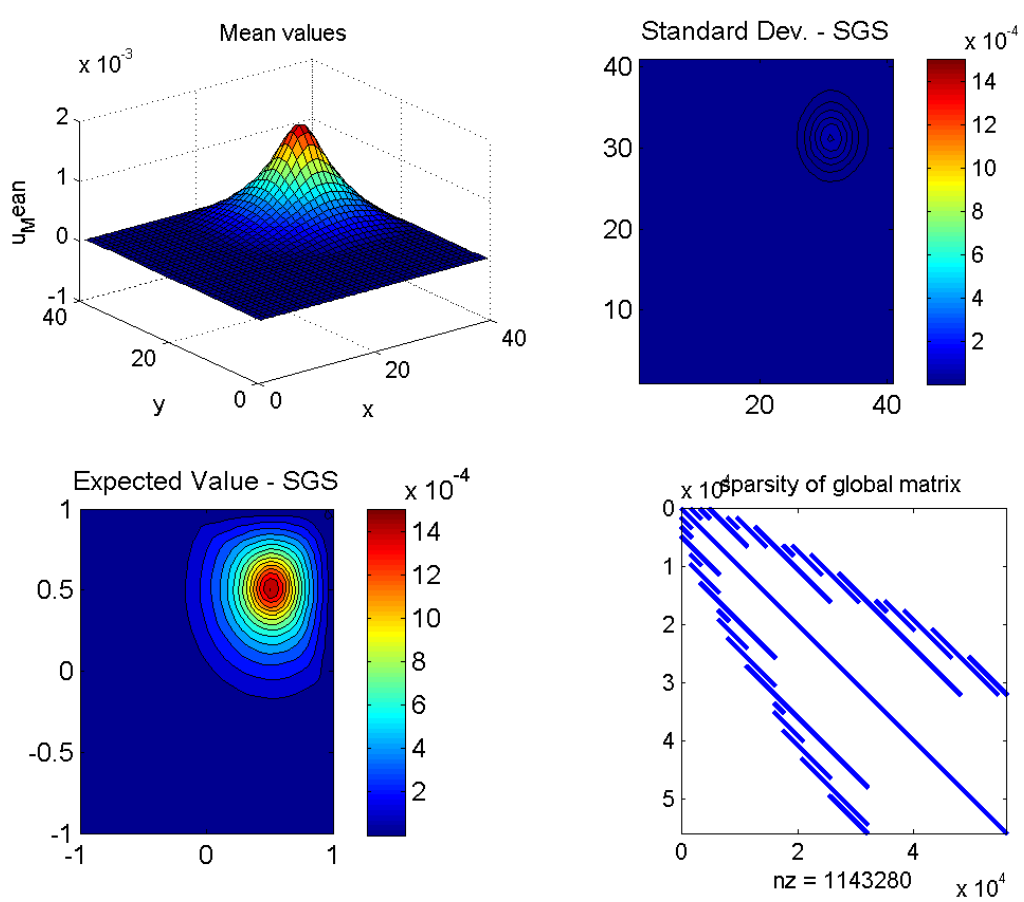

Fig 1.Illustration of the approximated solution and its mean value, standard deviation and global matrix sparsity for $\mathrm{d}=3, \mathrm{~N}=4,50 * 50$ mesh points in Example 1 .

Example 2: consider the following SPDE

$$
-\nabla \cdot(a(x, y, \omega) \nabla u(x, y, \omega))=z(x, y) \quad(x, y) \in D, \omega \in \Omega
$$

where $D=[-1,1]^{2}, \Omega \sim \mathrm{U}[-1,1]$, the boundary condition is given as

$$
u=1.1 \operatorname{sign}(\sin (\pi x) \sin (\pi y)) \text {. }
$$

and

$$
z(x, y)=\sin (\pi x) \sin (\pi y)+x .
$$

The random field $a(x, y, \omega)$ is characterized by its mean and covariance function

$$
E[a]=10, \quad R\left(x_{1}, x_{2}\right)=e^{-\left(x_{1}, x_{2}\right)}, \quad x_{1}, x_{2} \in[-1,1] .
$$

The truncated KLE of $a(x, y, \omega)$ can be expressed as:

$$
a(x, y, \omega)=10+\sum_{j=0}^{N} \sqrt{\lambda_{j}} \omega_{j} \phi_{j}(x) .
$$

The eigenpairs $\lambda_{j}, \omega_{j}$ in truncated KLE solve the integral equation

$$
\int e^{-\left(x_{1}, x_{2}\right)} \phi_{j}\left(x_{2}\right) d, x_{2}=\lambda_{j} \phi_{j}\left(, x_{1}\right)
$$

For this special case of the covariance function, we have explicit expression for $\lambda_{j}$ and $\phi_{j}$ [9].

Let $\omega_{j_{\text {even }}}$ and $\omega_{j_{\text {odd }}}$ solve the equations

$$
1-\omega_{j_{\text {even }}} \tan \left(\omega_{\text {jeven }}\right)=0, \omega_{j_{\text {odd }}}+\tan \left(\omega_{j_{\text {odd }}}\right)=0
$$


Then the even and odd indexed eigenfunctions are given by:

$$
\phi_{j_{\text {even }}}(x)=\frac{\cos \left(\left(\omega_{j_{\text {even }}} x\right)\right.}{\sqrt{1+\frac{\sin \left[\left(2 \omega_{\left.j_{\text {even }}\right)}\right)\right.}{2 \omega_{\text {even }}}}} \phi_{j_{\text {odd }}}(x)=\frac{\sin \left(\left(\omega_{j_{\text {odd }}} x\right)\right.}{\sqrt{1-\frac{\sin \left[\left(2 \omega_{j_{\text {odd }}}\right)\right.}{2 \omega_{j_{\text {odd }}}}}}
$$

and corresponding eigenvalues are given by:

$$
\lambda_{j_{\text {even }}}(x)=\frac{2}{\omega_{j_{\text {even }}}^{2}+1} \lambda_{j_{\text {odd }}}(x)=\frac{2}{\omega_{j_{\text {odd }}}^{2}+1}
$$

We choose $\omega_{j}=\left(\omega_{1}, \ldots, \omega_{N}\right)^{T}$ to be independent random variables uniformly distributed over the interval $[-1,1]$. First of all, we find numerical approximations of $\omega_{j_{\text {even }}}$ and $\omega_{j_{\text {odd }}}$ with bisection method, and then, with the eigenpairs evaluated with these $\omega_{j_{\text {even }}}$ and $\omega_{j_{\text {odd }}}$, we construct the KLE of $d=2, N=6$, which emphasis that $P=28$. Figure 2, illustrates mean value representation, standard deviation and expectation counter plot and global matrix sparsity, for $20 * 20$ mesh points
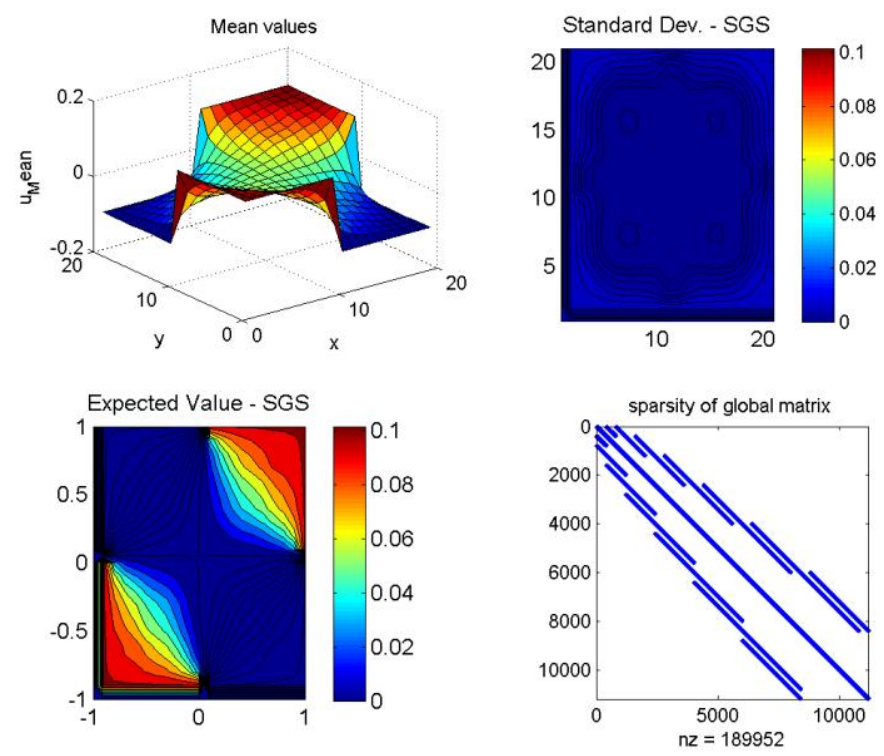

Fig 2. Illustration of the approximated solution and its mean value, standard deviation and global matrix sparsity for $\mathrm{d}=2, \mathrm{~N}=6,20 * 20$ mesh points in Example 2 .

Example 3: similar to Example 2, consider the following SPDE

$$
-\nabla \cdot(a(x, y, \omega) \nabla u(x, y, \omega))=z(x, y) \quad(x, y) \epsilon D, \omega \in \Omega
$$

where $D=[-1,1]^{2}, \Omega \sim \mathrm{U}[-1,1]$, with the boundary condition

$$
u(x, y, \omega)=0 \quad \text { on } D \times \partial \Omega
$$

and

$$
z(x, y)=2 \pi^{2} \sin (\pi x) \sin (\pi y) \quad \text { in } D \times \Omega
$$


Figure 3, illustrates mean value representation, standard deviation and expectation counter plot and global matrix sparsity, for 50*50 mesh points of spatial domain, $\mathrm{d}=1$, (number of random variables) and $\mathrm{N}=3$ (degree of polynomials) which emphasis that $\mathrm{P}=4$.
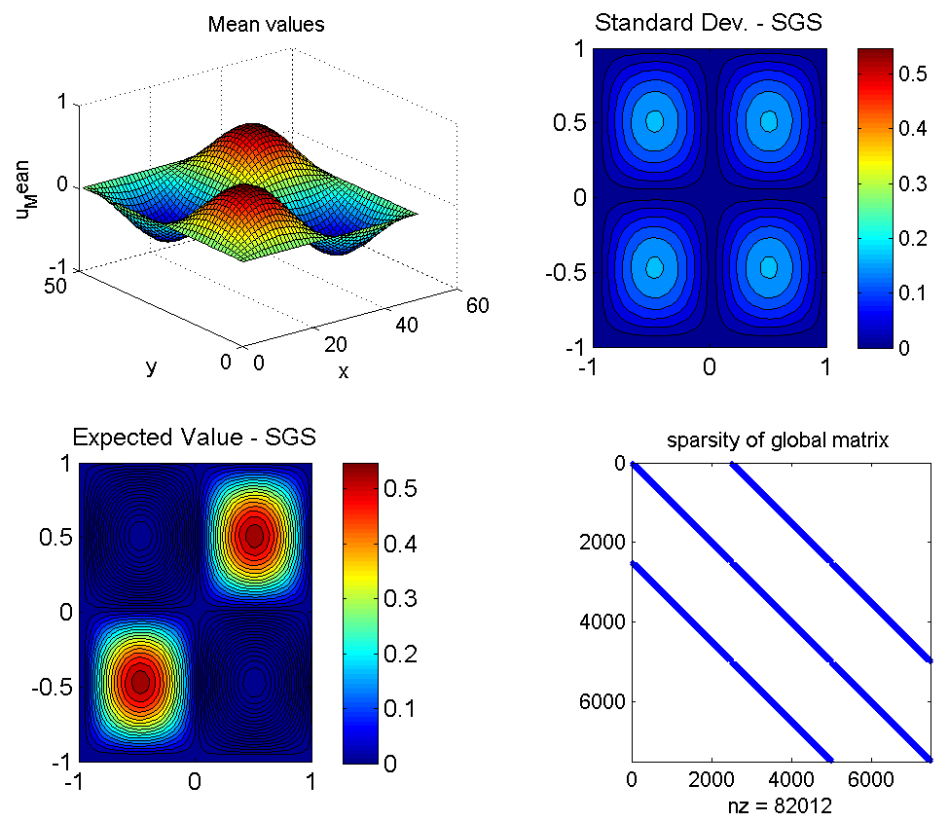

Fig 3. Numerical solution of example 3 with $50 * 50$ mesh points, $d=1, N=3$

\section{Conclusion}

We described a deterministic finite element (FE) solution algorithm for the stochastic elliptic boundary value problem, whose coefficient are assumed to be random field. Separation of random and deterministic variables is achieved via Karhunen-Loeve Expansion (KLE). Finite element discretization utilized in both spatial space and probability space. Using the Polynomial Chaos Expansion (PCE) and constructing the solution based on orthogonal basis and truncating the KLE of the permeability field lead to find a finite dimensional approximation. Finally, since the resulted system of equation governed by discretizing the problem is large and sparse, we use Kronecker product preconditioner and Newton's conjugate gradient method to solve this system of equation more accurate and fast. Numerical results presented for illustrating the theoretical results.

\section{References}

[1] I. Babuska, F. Nobile, and R. Tempone, A stochastic collocation method for elliptic partial differential equations with random input data, SIAM J. Numer. Anal., 45(3):1005-1034 (electronic), 2007.

[2] I. Babuska, R. Tempone, and G. E. Zouraris, Solving elliptic boundary value problems with uncertain coefficients by the finite element method: the stochastic formulation, Comput. Methods Appl. Mech. Engrg., 194 (12-16):1251-1294, 2005.

[3] I. Babuska, R. Tempone, and G. E. Zouraris, Galerkin finite element approximations of stochastic elliptic partial differential equations, SIAM J. Numer. Anal., 42(2):800-825 (electronic), 2004. [4] A. T. Bharrucha-Reid, On random operator equations in Banach space, Bull, Acad. Polon. 
Sci., Ser. Sci. Math. Astr. Phys. 7 (1959) 561-564.

[5] H. Elman, D. Furnival, Solving the stochastic steady-state diffusion problem using multigrid, IMA Journal of Numerical Analysis 27, 675-688, (2007).

[6] O. G. Ernst and E. Ullmann, Stochastic Galerkin matrices, SIAM J. Matrix Anal. Appl., 31(4):1848-1872, 2010.

[7] B. Fathi Vajargah, R. Asghari, Simulation Method for Solving Stochastic differential Equations with Constant Diffusion Coefficients, Journal of mathematics and computer Science 8 (2014) 28 -32.

[8] R. G. Ghanem, R. M. Kruger, Numerical solution of spectral stochastic finite element systems, Comput. Methods Appl. Mech. Engrg. 129 289-303, 1996.

[9] R. Ghanem, P.D. Spanos, Stochastic Finite Elements: A Spectral Approach, Springer, Berlin, 1991.

[10] M. heydari, M. Mahdavi Mazdeh and M. Bayat, Stochastic Online Scheduling With Preemption Penalties, Journal of mathematics and computer Science 6 (2013), 238-250

[11] T. Hisada, S. Nakagiri, Role of the stochastic finite element method in structural safety and reliability, Proc. 4th Int. Conf. on Struct. Safety and Reliability, Kobe, Japan, vol. I, 385-394, 1985,.

[12] K. Karhunen, Uber lineare Methoden in der Wahrscheinlichkeitsrechnung, Ann. Acad. Sci. Fennicae. Ser. A. I. Math.-Phys., 1947(37):79, 1947.

[13] M. Kleiber, T.D. Hien, The Stochastic Finite Element Method, Wiley, New York, 1992.

[14] W. K. Liu, T. Belytschko, A. Mani, Random field finite elements, Int. J. Num. Meth. Engrg. 23 1831-1845, 1986.

[15] M. Lo“eve, Fonctions aleatoires de second ordre, Revue Sci., 84:195-206, 1946.

[16] S. Nakagiri, T. Hisada, Stochastic finite element method applied to structural analysis with uncertain parameters, Proc. Intl. Conference on FEM, August pp. 206-211, 1982.

[17] J. Nocedal and S. J. Wright, Numerical Optimization, Springer Verlag, Berlin, Heidelberg, New York, second edition, 2006.

[18] M. Shinozuka, E. Lenoe, A probabilistic model for spatial distribution of material properties, Eng. Fract. Mech. 8 217-227, 1976.

[19] P.D. Spanos, R. Ghanem, Stochastic finite element expansion for random media, Eng. Mech., ASCE $115,1989$.

[20] E. Ullmann, A Kronecker product preconditioner for stochastic Galerkin finite element discretizations, 32(2):923-946, 2010.

[21] E.H. Vanmarcke, M. Grigoriu, Stochastic finite element analysis of simple beams, J. Eng. Mech., ASCE 109 (1983) 1203-1214.

[22] N. Wiener, The homogeneous chaos, Amer. J. Math., 60:897-936, 1938. 